\title{
Hunter Syndrome
}

National Cancer Institute

\section{Source}

National Cancer Institute. Hunter Syndrome. NCI Thesaurus. Code C61260.

An X-linked, inherited lysosomal storage disease caused by the deficiency of the enzyme iduronate sulfatase that is responsible for the degradation of mucopolysaccharides. It nearly always affects males and is characterized by the accumulation of mucopolysaccharides in various organs, resulting in mental dysfunction, enlarged abdomen, hearing loss, obstructive airway disease, heart disease, and hepatosplenomegaly. 